\title{
Análisis y evaluación de aplicaciones para desarrollar la comunicación en el alumnado con trastorno del espectro autista
}

\author{
Analysis and evaluation of applications focused on developing communication skills in \\ students with autism spectrum disorder
}

\author{
María Capel Sánchez \\ macasan96@gmail.com
}

Universidad de Murcia (España)

\section{Resumen}

La tecnología de ayuda en la educación de las personas con autismo es esencial para desarrollar su comunicación. Actualmente hay un gran aumento de aplicaciones digitales educativas que resultan ser muy interesantes para ellos, aunque se debe identificar su calidad con los instrumentos adecuados. Por ello, los propósitos generales de la investigación son conocer la situación actual del papel que tienen las tecnologías educativas en la intervención educativa en los estudiantes con autismo y analizar y evaluar qué programas resultan más adecuados para desarrollar su comunicación.

En esta investigación de tipo descriptivo se realiza un análisis y evaluación de 50 aplicaciones digitales educativas que desarrollan la comunicación en el alumnado que presenta autismo. La recogida de información y análisis de dichas aplicaciones se llevan a cabo por medio de una rúbrica.

Los resultados mostraron que existen 8 aplicaciones con una calidad excelente y 17 con una puntuación adecuada, recomendándose así su uso en intervenciones educativas. Asimismo, se realizó un catálogo con las aplicaciones seleccionadas como recurso útil para docentes y familias.

Palabras clave: trastorno del espectro autista; apps; comunicación; evaluación de calidad; rúbrica.

\begin{abstract}
Technology is essential to help with the development of communication skills in people who have autism. Currently, there has been a significant increase in the number of applications which turn out to be very interesting for them, however its quality must be identified with proper tools. Therefore, the general purpose of this investigation is to determine the current state of educative technologies' role on the educative intervention in students with autism and analyse and evaluate which software are more adequate to develop their communicative skills.

In this descriptive investigation, 50 educative applications focused on developing communication skills in students with autism are analysed and evaluated. The information and analysis have been carried out by means of a rubric.

Results showed that 8 applications had excellent quality and 17 with a decent score, allowing me to recommend their use in educative interventions. Additionally, an application catalogue has been developed, which may be of use to teachers and families.
\end{abstract}

Keywords: autism spectrum disorder; apps; communication; quality evaluation; rubric. 


\section{INTRODUCCIÓN}

La sociedad del ahora ofrece multitud de información y campañas de sensibilización y concienciación sobre las personas con trastorno del espectro autista (en adelante TEA), no obstante, sigue existiendo un gran desconocimiento sobre este trastorno (Terrazas et al., 2016). Los estudiantes con TEA presentan alteraciones en la reciprocidad social, en la capacidad simbólica y conducta imaginativa, en el sentido de la actividad e intereses y en la comunicación verbal y no verbal (Wing, 1998). En relación con sus déficits en las habilidades comunicativas, es de vital importancia conocer que necesitan el apoyo de Sistemas Aumentativos y Alternativos de Comunicación, considerando que existe una gran heterogeneidad en el perfil comunicativo y lingüístico de cada niño con TEA (Montero, 2019).

En muchas ocasiones, debido a las características que presentan los niños con autismo, se producen situaciones de exclusión en su centro, es por ello que, hoy en día, las tecnologías son un apoyo muy importante para facilitar su propio aprendizaje (Pastor et al., 2014). Además, se está adquiriendo mucha importancia en la inclusión de la tecnología educativa en el proceso de enseñanza-aprendizaje en niños con autismo (García et al., 2016), ocupando estas un papel relevante a la hora de atender a sus necesidades educativas.

Actualmente, se ha contemplado un aumento considerable de la tecnología de apoyo para el alumnado con TEA, especialmente en aplicaciones digitales (apps) y programas, como consecuencia de los dispositivos móviles como tabletas y smartphones (Sanromà et al., 2017). Estos dispositivos mejoran la calidad de vida y muestran una diversidad de posibilidades y opciones para la intervención educativa de las personas con TEA, además de que facilitan el acceso a la información en cualquier momento y presentan gran flexibilidad y adaptación (Jiménez et al., 2017). Por este motivo, el desarrollo de la tecnología móvil ha conllevado nuevas herramientas y oportunidades de comunicación para los niños con TEA (McNaughton y Light, 2013; Boyd et al., 2015).

Larco et al. (2018) mencionan que, en las aplicaciones para niños con autismo las competencias menos desarrolladas son las vinculadas con la comunicación con un 33\%, siendo las principales competencias la autonomía, las habilidades sensoriomotoras y las sociales con un 63\%. Asimismo, añaden que el $8 \%$ de las aplicaciones se centran en la competencia comunicativa, un $49 \%$ en el medio ambiente y un $38 \%$ en la autonomía, habilidades sensoriomotoras y sociales. Por su parte, señalan como futuras líneas de investigación la búsqueda de las razones por las que no hay aplicaciones para desarrollar y potenciar el lenguaje y la competencia de comunicación lingüística.

Por otro lado, diversos autores indican en sus investigaciones que el gran número de aplicaciones digitales educativas supone un problema, debido a que, en muchas ocasiones, los docentes y familias las usan de forma inapropiada, seleccionándolas sin ser analizadas y/o según la valoración de los usuarios (Ok et al., 2015; Weng y Taber-Doughty, 2015; Bouck et al., 2016). Destacan que la puntuación de una aplicación en los grandes mercados (Apple Store o Google Play) es deficiente e inapropiada, subrayando que deben ser analizadas de manera exhaustiva utilizando instrumentos de evaluación específicos que evalúen su calidad y 
capacidad para desarrollar las necesidades del alumnado. De ahí que hayan surgido investigaciones que faciliten a los profesionales de la educación y familias, catálogos de aplicaciones calificadas y rúbricas para realizar una selección adecuada en función del perfil del alumno (Ok et al., 2015; Weng y Taber-Doughty, 2015; Bouck et al., 2016; Cepeda et al., 2017; Larco et al., 2018).

A raíz de estos problemas, la finalidad del estudio que se presenta en este artículo, consiste en la evaluación de aplicaciones digitales para el desarrollo de la comunicación en el alumnado con TEA, con el fin de ofrecer a los profesionales de la educación un catálogo con la calificación de cada una de ellas, así como una rúbrica adaptada al castellano de diversas investigaciones internacionales. Para su logro, se plantean los siguientes objetivos generales y específicos:

OG1. Analizar y evaluar qué programas digitales resultan más adecuados en la intervención educativa del alumnado con TEA para desarrollar la comunicación.

OE-1.1. Buscar y seleccionar rúbricas de evaluación de aplicaciones digitales educativas para alumnado con necesidades educativas especiales.

OE-1.2. Explorar y evaluar programas digitales para desarrollar la comunicación en el alumnado con Trastorno del Espectro Autista.

OE-1.3. Diseñar y crear un catálogo de aplicaciones que desarrollen la comunicación en el alumnado con TEA.

\section{MÉTODOS}

\subsection{Diseño metodológico}

La investigación se fundamenta en un diseño metodológico con enfoque cuantitativo no experimental de tipo descriptivo. Se ha elegido este tipo de enfoque puesto que es el más adecuado para la obtención de los datos que se quieren analizar.

Los autores Hernández-Sampieri et al. (2014) expresan que con los estudios de alcance descriptivo "se busca especificar las propiedades, las características y los perfiles de personas, grupos, comunidades, objetos o cualquier otro fenómeno que se someta a un análisis" (p.92). Indudablemente, para implementar una investigación descriptiva, se debe realizar una búsqueda documental sobre los antecedentes del tema para evitar repeticiones, por lo que el estudio descriptivo incluye la investigación documental (Salinas, 2012). 


\subsection{Muestra}

La muestra de la investigación está formada por 50 aplicaciones que desarrollan la comunicación en el alumnado con autismo. El procedimiento utilizado para su selección ha sido no probabilístico de tipo intencional, dado que se seleccionarán los elementos dependiendo de las características del estudio y objetivos propuestos (Hernández-Sampieri et al., 2014). Para ello, se ha realizado una exploración sistemática en diversas plataformas especializadas, investigaciones y buscadores, siendo los términos de búsqueda: "aplicaciones para desarrollar la comunicación en niños con autismo", "aplicaciones y TEA", "software y TEA" y "Apps para la comunicación y TEA". Finalizado el análisis, se seleccionaron 148 programas y, entre ellos, se siguieron los criterios de inclusión/exclusión expuestos en el siguiente flujograma:

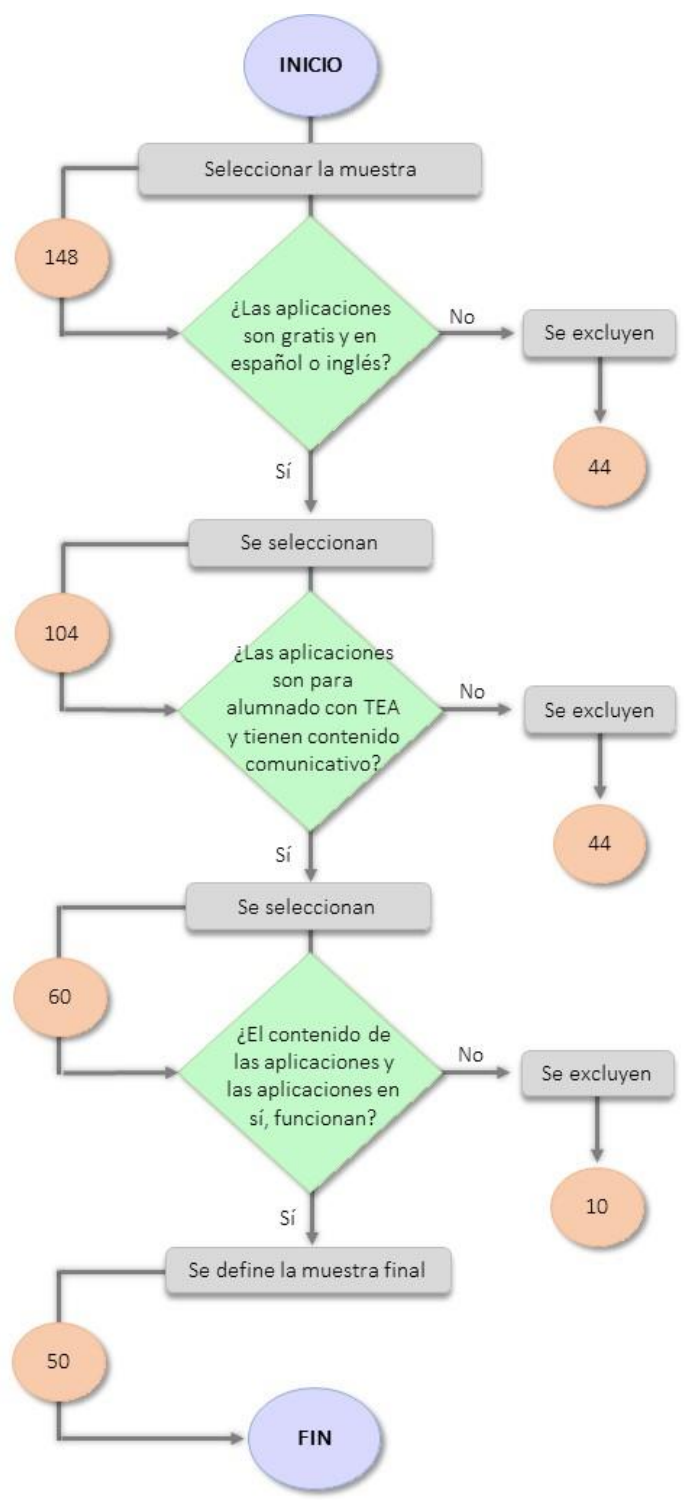

Figura 1. Flujograma del proceso de selección de la muestra 
Las 50 aplicaciones seleccionadas en la última fase son las que se señalan en la siguiente tabla:

Tabla 1. Aplicaciones seleccionadas para su análisis y evaluación

\begin{tabular}{llll}
\hline & & & Aplicaciones \\
\hline 2 & In TIC TEA & 26 & Talk Autismo Imagen Discusión \\
\hline 3 & Día a Día & 27 & Proyect@ SAAC \\
\hline 4 & DictaPicto & 28 & Nikitalk \\
\hline 5 & e-Mintza & 29 & Aarlens \\
\hline 6 & Cuentos visuales: José aprende & 31 & Piktoplus - ACC speech, Education, Autism and much more \\
\hline 7 & School Adapter & 32 & Autism Help \\
\hline 8 & Sígueme & 33 & Words in pictures \\
\hline 9 & \#Soyvisual & 34 & PictogramAgenda \\
\hline 10 & Pictotraductor & 35 & Comunicador \\
\hline 11 & Pictosonidos & 36 & Pictogram Agenda \\
\hline 12 & Pictocuentos & 37 & OTTAA proyect \\
\hline 12 & Pictoeduca & 38 & Jugando con las vocales \\
\hline 14 & WikiPicto & 39 & ISay \\
\hline 15 & Programa Araword & 40 & LetMeTalk: Talker SAAC, CAA, SAC \\
\hline 16 & Conversation therapy lite & 41 & AbaPlanet Lite \\
\hline 17 & Picaa 2 & 42 & PictoTEA \\
\hline 18 & Autismo iHelp & 43 & Háblalo \\
\hline 19 & LeoconLula & 44 & Picto One: TEA - Comunicación con pictogramas \\
\hline 20 & Las Pelusas & 45 & Terapia del Lenguaje y Cognitiva con MITA \\
\hline 21 & El Pajarito Rosa & 46 & Talk UP! Autismo Communicator \\
\hline 22 & Pictoagenda & 47 & Helpmetalk \\
\hline 23 & HablaFácil Autismo: Diegodice & 48 & Autism early intervetion \\
\hline 24 & Avaz AAC & 49 & Voice - AMIKEO APPS \\
\hline 25 & CommBoards & 50 & SymboTalk - AAC Talker \\
\hline
\end{tabular}

\subsection{Instrumento de recogida de datos}

Para la obtención de la información del estudio, se ha realizado una rúbrica que parte de la exploración y análisis de 8 rúbricas validadas. Para ello, se ha tenido en cuenta su costo, disponibilidad y facilidad de uso y que la información que se vaya a obtener sea lo más detallada, precisa y objetiva posible para conseguir los propósitos planteados. En la Tabla 2 se exponen las que han sido analizadas para la adaptación del instrumento de recogida de datos de esta investigación. 
Tabla 2. Rúbricas seleccionadas para su análisis

\begin{tabular}{cc}
\hline \multicolumn{1}{c}{ Autor } & Rúbrica \\
\hline Marqués (1999) & Espacios web de interés educativo. Ficha de identificación y evaluación \\
\hline Higgins et al. (2000) & Components to be considered in all types of software. \\
\hline Walker (2010) & Evaluation Rubric for iPod Apps \\
\hline $\begin{array}{c}\text { More y Travers } \\
\text { (2013) }\end{array}$ & Preschool App Evaluation Guide \\
\hline $\begin{array}{c}\text { Weng y Taber- } \\
\text { Doughty (2015) }\end{array}$ & App Evaluation Rubric \\
\hline Ok et al. (2015) & Rubric for evaluating iPad apps to improve communication skills of students with \\
\hline Boyd et al. (2015) & autism spectrum disorder \\
\hline Cepeda et al. (2017) & Guía para el análisis de materiales didácticos digitales \\
\hline
\end{tabular}

Una vez examinadas las rúbricas, el siguiente paso fue elegir aquellas cuyo propósito era evaluar aplicaciones para alumnado con necesidades educativas especiales (NEE). EI instrumento final ${ }^{1}$ realizado abarca las rúbricas desarrolladas por Weng y Taber-Doughty (2015), Ok et al. (2015) y Boyd et al. (2015), seleccionando de cada una de ellas las secciones, categorías y propiedades que más se adaptan a la investigación. En primer lugar, se tradujeron las tres rúbricas, y a partir de aquí se seleccionaron las categorías y secciones más idóneas de cada una.

\section{RESULTADOS}

Para el análisis de datos y su mejor obtención de información, se ha diseñado una hoja de $E x c e l^{2}$ en la cual se presentan las 50 aplicaciones evaluadas con sus respectivas rúbricas.

\subsection{Información básica de las aplicaciones}

De las aplicaciones seleccionadas, 18 son de Android e iOS, 18 de Android, 7 de iOS, 6 de web y 5 de escritorio. Tal y como se puede apreciar existe una gran diferencia entre el número de aplicaciones analizadas de Android e iOS con las de web y de escritorio.

Por otra parte, hay un $13 \%$ de las aplicaciones que están en versión Lite, suponiendo esto ciertas limitaciones; $y$, un $74 \%$ son en versión Completa, lo que permite que se puedan utilizar

\footnotetext{
${ }^{1}$ Rúbrica realizada de los autores Weng y Taber-Doughty (2015), Ok et al. (2015) y Boyd et al. (2015): https://drive.google.com/file/d/108z9q87CTY4BWUiPqUdzLtcsQPOSaZWN/view? usp=sharing

${ }^{2}$ Hoja de Excel del análisis y evaluación de las 50 aplicaciones:

https://drive.google.com/open?id=1T4qMUzYnZz8e9Mvv2vEw4T7yjpai7alo
} 
sin pagos dentro de la aplicación. En el total de las aplicaciones no se ha encontrado ninguna en versión Pro, es por ello que no se pueden comparar con las demás versiones.

\subsection{Evaluación del diseño y características técnicas de las aplicaciones}

En este apartado se hará alusión a los resultados generales de la Sección 2, donde se incluyen las puntuaciones (1), (2) o (3) de cada categoría evaluada de la muestra total seleccionada. Para facilitar su descripción, se han realizado tres gráficos de columnas en los cuales se analiza la información obtenida de las categorías en porcentajes. Cada uno de los gráficos contiene 6 categorías descritas, siendo el total 18.

Si se observa la Figura 3, en la primera categoría Objetivo, la máxima puntuación (3) tiene el $98 \%$ del total y el $2 \%$ restante es para la calificación media (2), obteniendo así resultados excelentes en esta categoría del total de las aplicaciones. Además, en la categoría Estrategia, sigue siendo relevante la calificación más alta (3) con un $84 \%$ y el $16 \%$ con una puntuación inferior (2). En la categoría Ejemplos, se aprecia que la calificación máxima (3) sigue siendo relevante, pero disminuye con un 50\% del total, casi igualándose con la puntuación inferior (2) con un 44\%. También, se aprecia que aparece la calificación más baja (1) inexistente en las anteriores categorías con un $6 \%$ del total. La categoría Práctica también destaca por su calificación máxima (3) con un 86\%, una calificación media (2) con un $10 \%$ y una puntuación más baja (1) con un $4 \%$.

En contraposición con estas cuatro categorías mencionadas, se puede observar que en las dos siguientes, el porcentaje de su calificación máxima (3) pasa a disminuir, aumentando considerablemente la puntuación mínima (1). En concreto, la categoría Corrección de errores y retroalimentación tiene un $14 \%$ de la puntuación más alta (3) y un $86 \%$ de la puntuación mínima (1). Asimismo, la categoría Análisis de errores tiene la puntuación más alta (3) con un 10\% y la puntuación más baja (1) con el $90 \%$. 


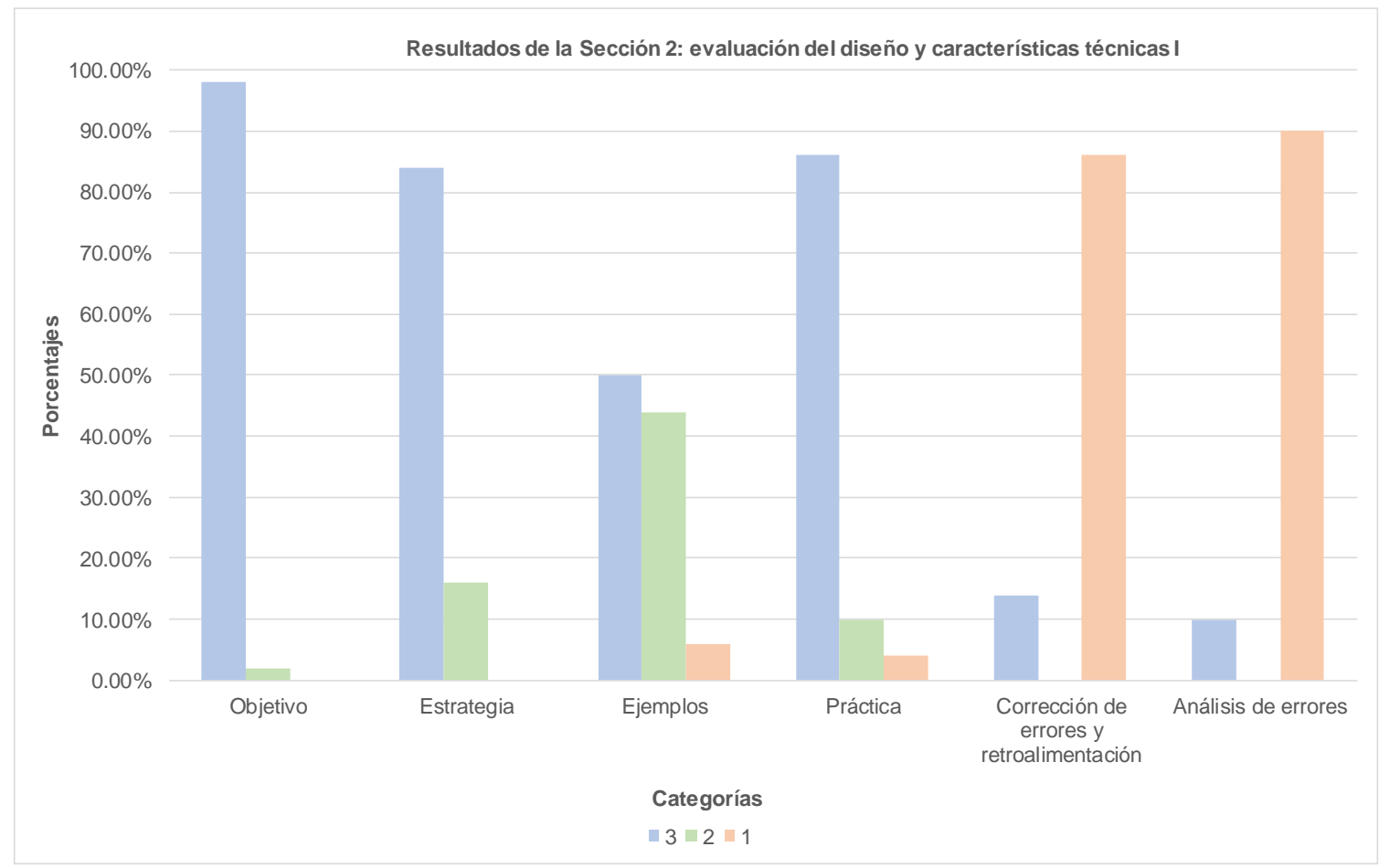

Figura 3. Resultados de la Sección 2: evaluación y características técnicas I.

Siguiendo con el análisis, si observamos la Figura 4, se puede observar que la puntuación mínima (1) sigue siendo destacable como en las dos categorías anteriores. Se trata de la categoría Control del progreso, la cual presenta un $20 \%$ en la calificación más alta (3), un $2 \%$ en la media (2) y un $78 \%$ en la más baja (1). En las cuatro siguientes categorías, se aprecia un notable aumento de porcentajes de la máxima puntuación (3). En la categoría Motivación, el porcentaje con la máxima puntuación (3) es del 70\%, la puntuación media (2) con un 20\% y con la puntuación mínima (1) con un $10 \%$. Por otra parte, la categoría Usabilidad presenta un 70\% con la máxima puntuación (3), un $2 \%$ con la puntuación media (2) y un $28 \%$ con la puntuación inferior (1), aumentando esta con respecto a la anterior. Asimismo, en la categoría Navegación, se aprecia una desescalada en cuanto a sus calificaciones, un 66\% a la puntuación más alta (3), un $30 \%$ a la puntuación media (2), y un $4 \%$ a la calificación más baja (1). En relación con la categoría Estímulos visuales y auditivos, se observa el alto porcentaje con la calificación más alta (3) con un $92 \%$, un $6 \%$ con una calificación media (2) y un $2 \%$ con la calificación más baja (1). La última categoría de esta figura: Habilidades motoras, se percibe el mayor porcentaje con la puntuación media (2) con un 54\% y porcentajes igualados en las demás calificaciones, específicamente, $24 \%$ con la puntuación superior (3) y un 22\% con la calificación más baja (1). 


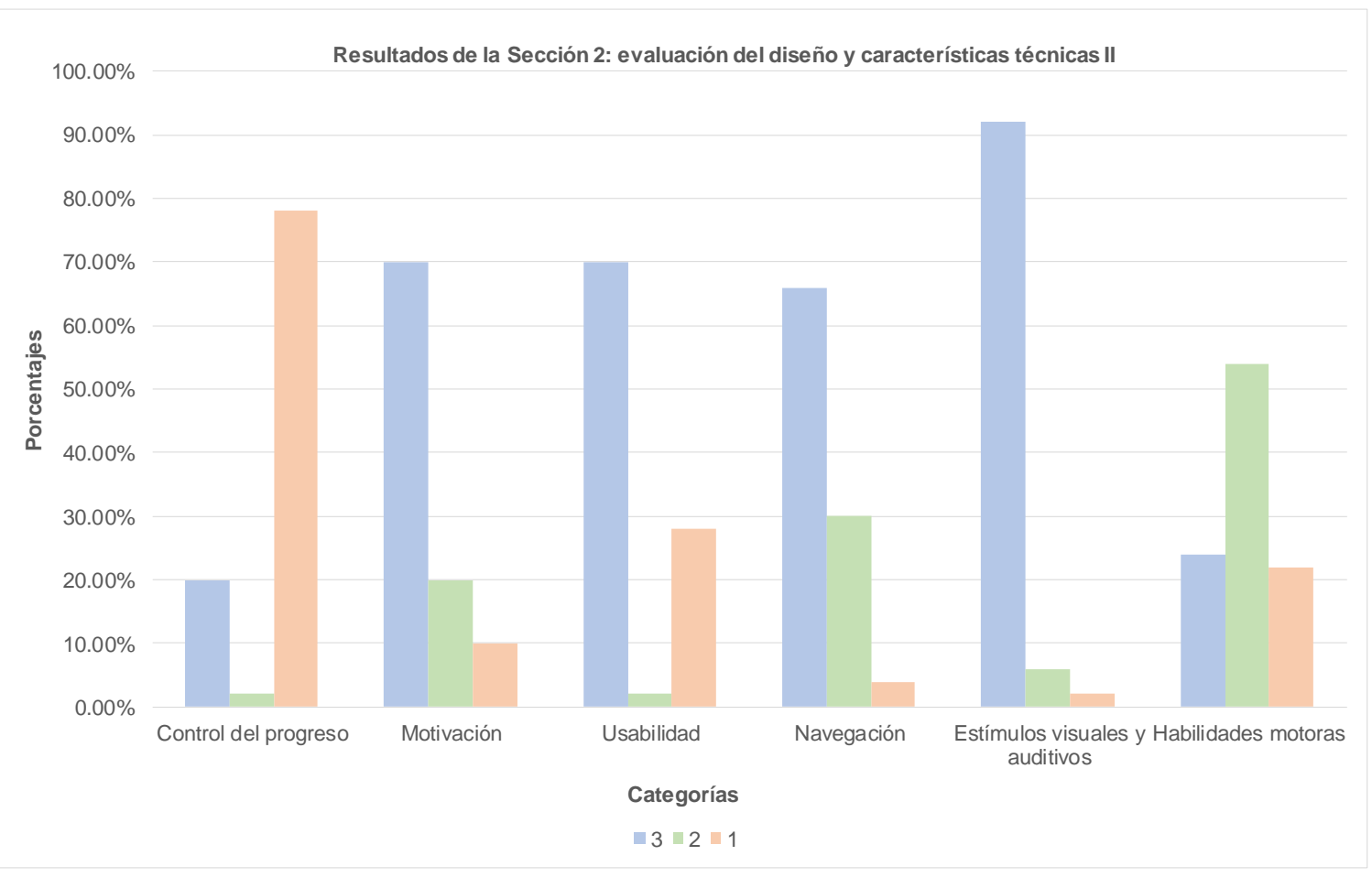

Figura 4. Resultados de la Sección 2: evaluación del diseño y características técnicas II.

En relación al siguiente gráfico (Figura 5), se contempla una categoría denominada Configuraciones personalizadas que se compone de cuatro subcategorías: General, Vocabulario y Gramática, Fuente y Fotografías. En la primera, se aprecian porcentajes más igualados: 28\% con la puntuación máxima (3), 40\% con la puntuación media (2) y un $58 \%$ con la puntuación más baja (1). En la subcategoría Vocabulario y gramática se observa un decrecimiento en la calificación media (2) con un 4\% y un aumento en la calificación máxima (3) del 58\%, y con la calificación más baja (1) con un 38\%. En la tercera subcategoría Fuente, se vuelven a equilibrar los porcentajes, un $44 \%$ a la máxima puntuación (3), un 24\% a la puntuación media (2) y un $32 \%$ a la puntuación inferior (1). Por último, en la subcategoría Fotografías se distingue un porcentaje más elevado con la calificación máxima (3) con un $56 \%$, un $36 \%$ a la calificación más baja (1) y con un $8 \%$ a la calificación media (2).

Por otra parte, en esta figura se aprecian otras dos categorías, donde sus porcentajes más elevados están vinculados a la máxima puntuación (3), presentando porcentajes menores en la calificación media (2) e inexistentes en la calificación más baja (1). En concreto, la categoría Error de contenido y sesgo, presenta un $82 \%$ en la calificación más alta (3) y un $18 \%$ en la calificación media (2), así como la categoría Manual/Tutorial un 74\% a la puntuación más alta (3) y un $26 \%$ a la media (2). 


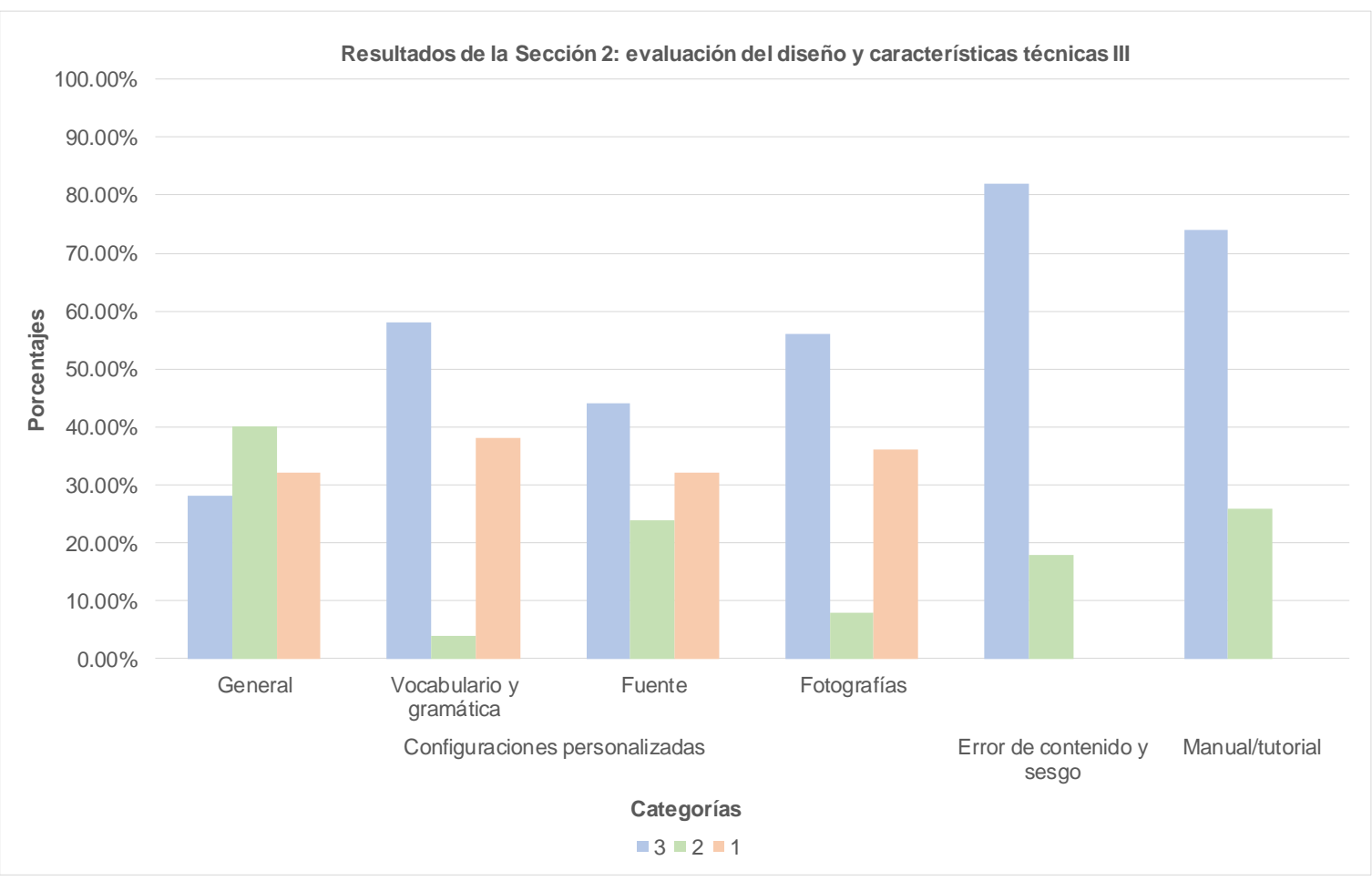

Figura 5. Resultados de la Sección 2: evaluación del diseño y características técnicas III.

\subsection{Impresiones generales de las aplicaciones}

Esta sección de la rúbrica se ejecuta una vez evaluadas las categorías de la sección dos, dado que se puede tener una perspectiva general de la aplicación. Si examinamos la Figura 6, se observa que, en general, los resultados obtenidos son positivos, puesto que las tres categorías indicadas tienen porcentajes elevados en la calificación más alta (3). La primera de ellas tiene un $82 \%$ en la máxima (3), un $14 \%$ en la media (2) y un $4 \%$ en la más baja (1). La segunda presenta un $86 \%$ con la puntuación más alta (3), un $8 \%$ con una media (2) y un $6 \%$ con la más baja (1). Por último, se destaca en la última categoría el 100\% con la calificación más alta (3). Esto es causa de que todas las aplicaciones seleccionadas son gratuitas. 


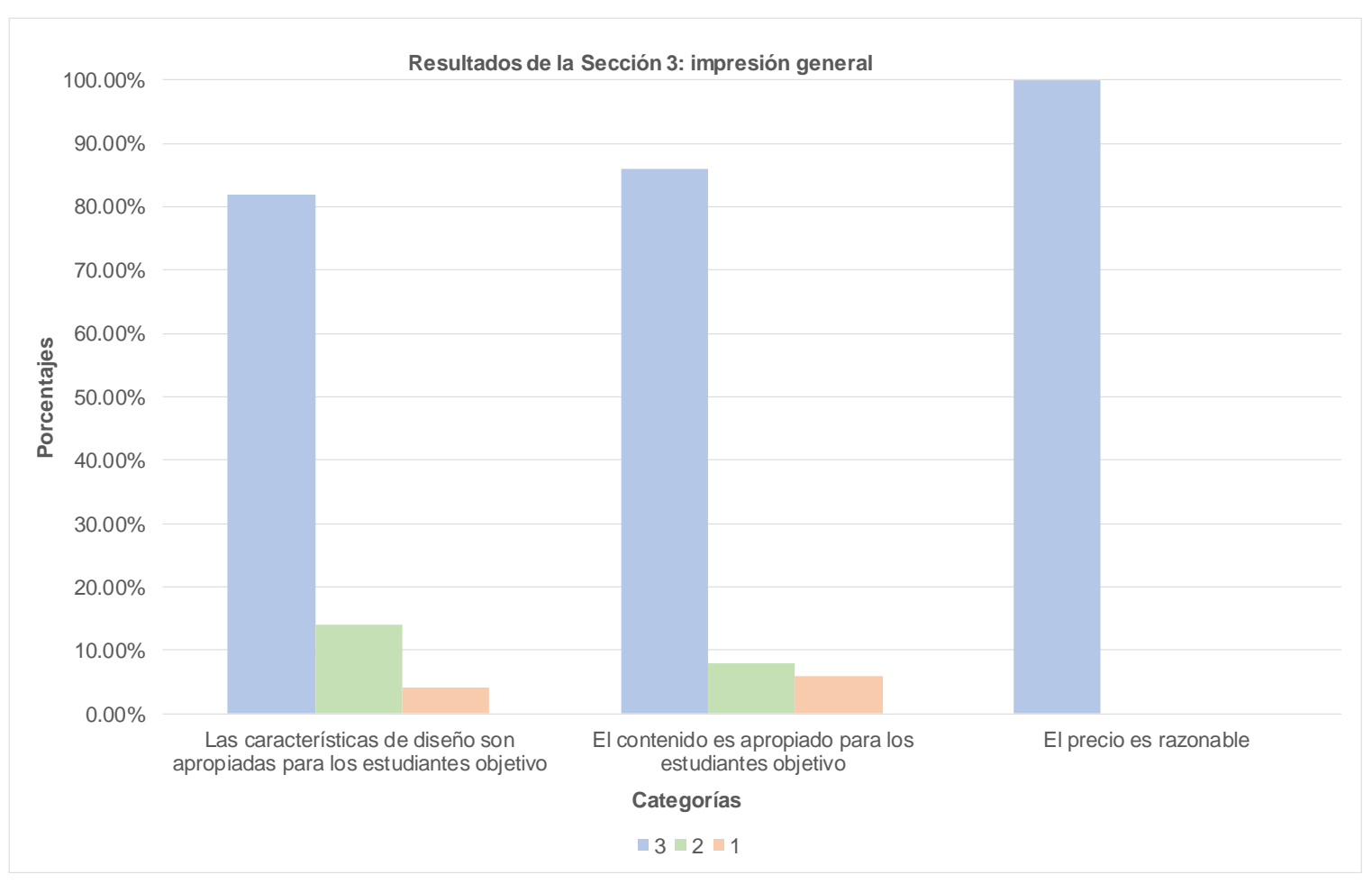

Figura 6. Resultados de la sección 3: impresión general.

\subsection{Resultados del estudio correlacional}

En este bloque se realizará un estudio correlacional entre las valoraciones medias de los usuarios en los mercados más relevantes, mi valoración rápida como experta tras el análisis previo realizado y la calificación final de la aplicación. Para ello, se analizarán las variables mencionadas con la ayuda de los siguientes gráficos de dispersión, teniendo en cuenta que la recogida de datos de la Figura 7 se realizará con la muestra total, en cambio, la Figura 8 y 9 se tendrán en cuenta 39 aplicaciones del total, pues 11 de las aplicaciones evaluadas no han sido valoradas por los usuarios. 


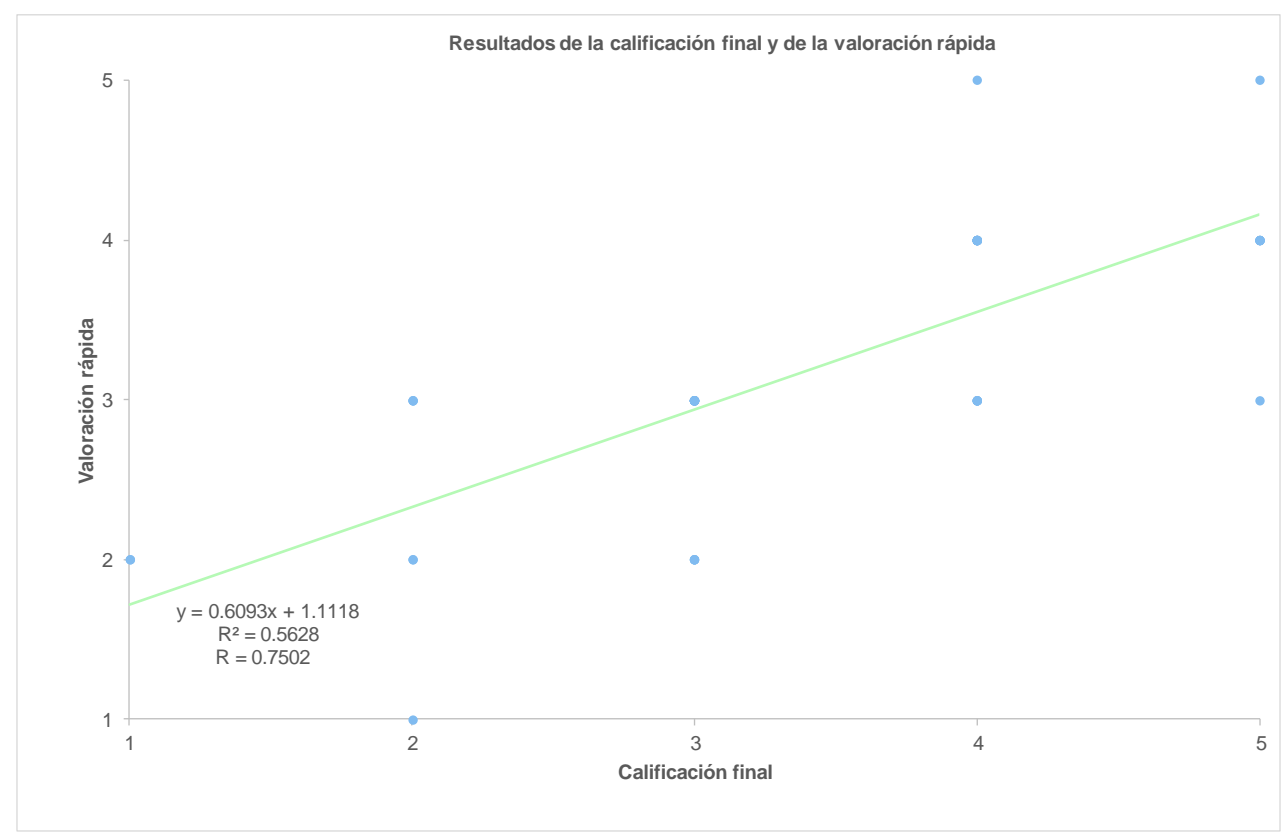

Figura 7. Resultados de la calificación final y de la valoración rápida.

La línea de tendencia de la Figura 7, la cual establece el comportamiento medio entre las variables de calificación final y de valoración rápida, indica que la relación entre ellas es lineal ascendente y están correlacionadas. El resultado del coeficiente de correlación $(r)$ es del 0.7502, lo que expresa que tienen una relación positiva considerable, a su vez, lo mismo ocurre con el coeficiente de determinación $\left(r^{2}\right)$, puesto que es más próximo al 1 que al 0 , por lo que el modelo se ajusta adecuadamente y podemos afirmar que las variables están correlacionadas.

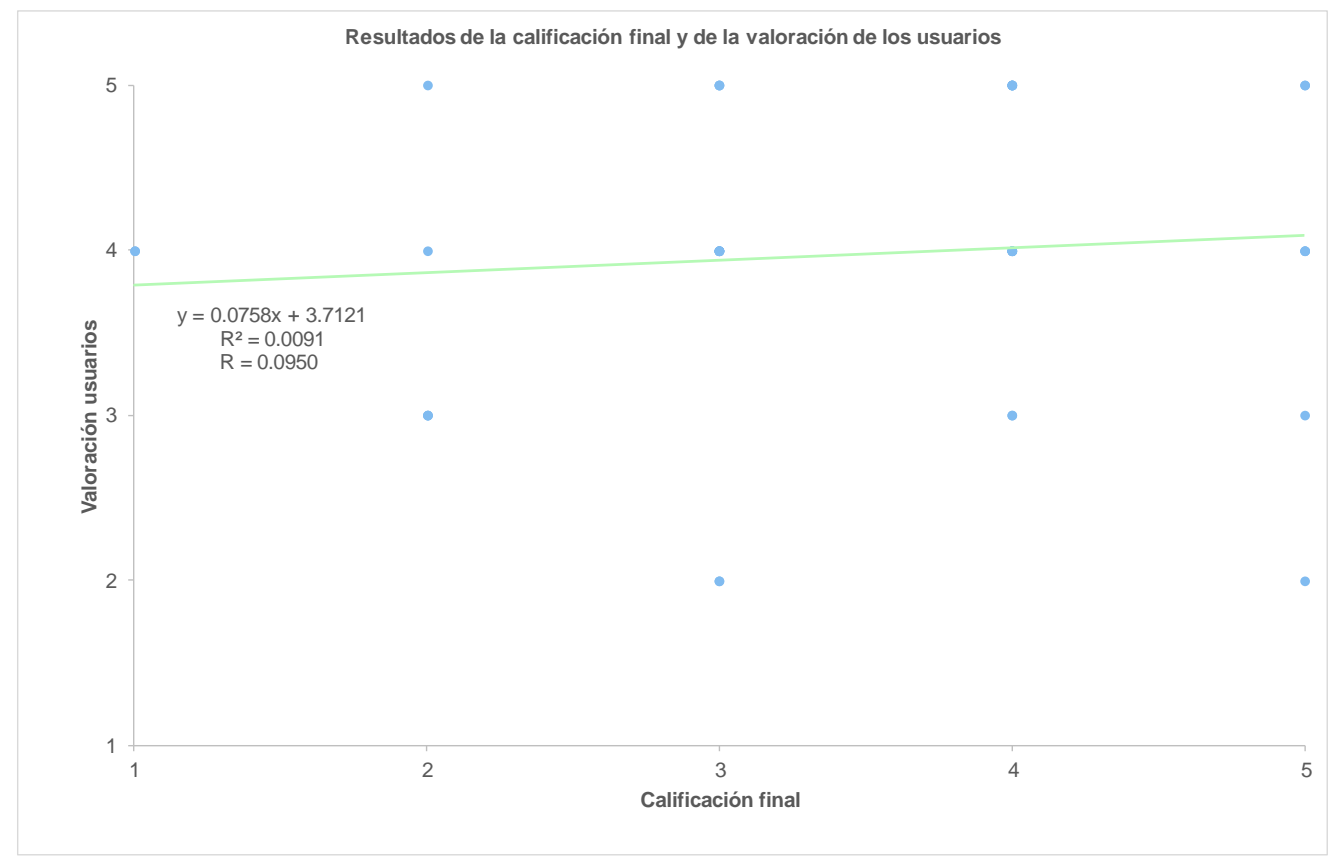

Figura 8. Resultados de la calificación final y de la valoración de los usuarios. 
En cambio, en la Figura 8 se puede inferir lo contrario a la Figura 7, debido a que las variables de valoración de los usuarios y de calificación final no tienen ninguna correlación. Este hecho se puede observar en el coeficiente de correlación $(r)$ y en el coeficiente de determinación $\left(r^{2}\right)$ debido a que son muy débiles. Además, los datos del gráfico se perciben muy distantes entre sí y en relación a la línea de tendencia. Por todo ello, se puede concluir que la correlación entre las dos variables es nula.

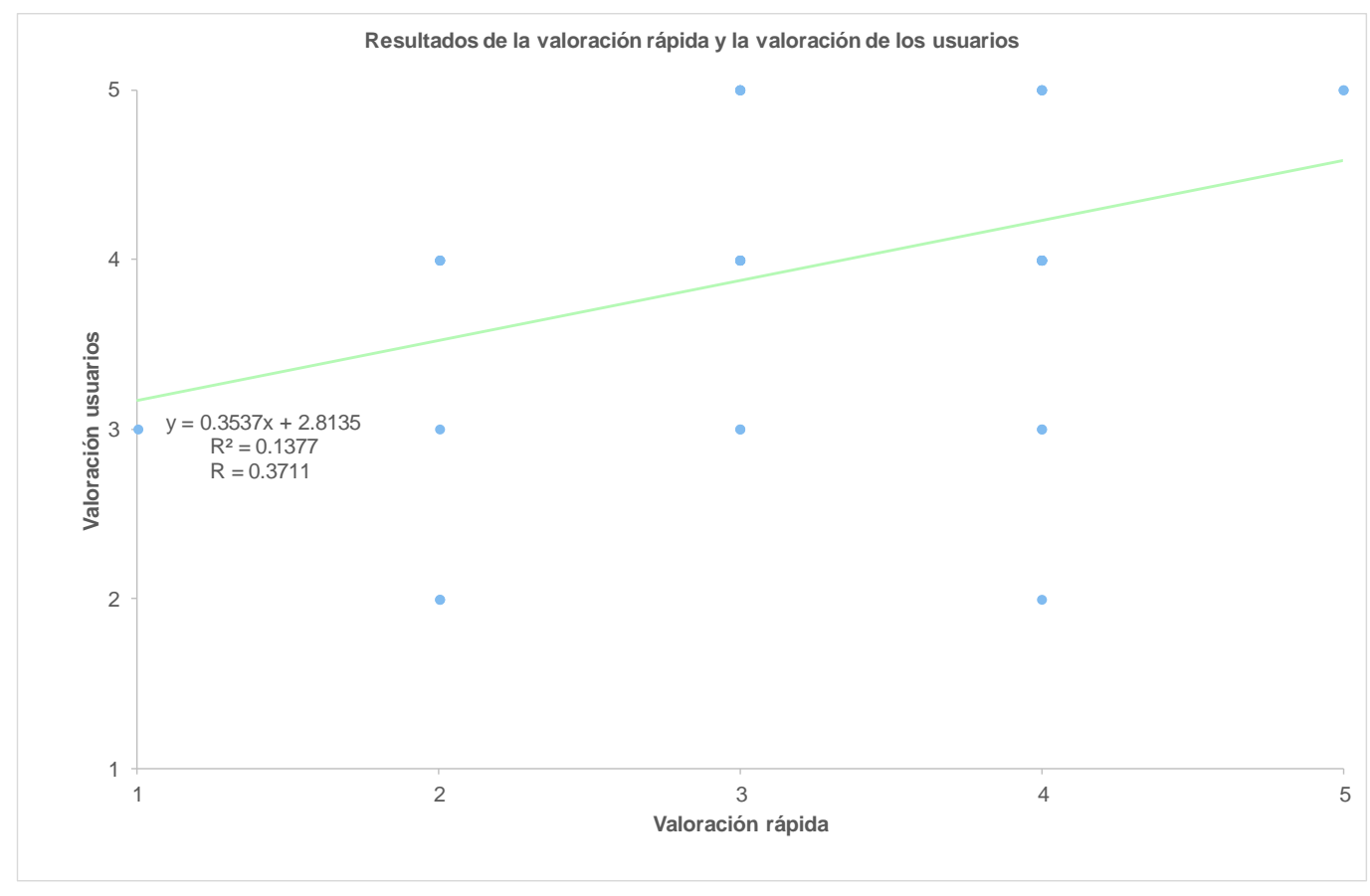

Figura 9. Resultados de la valoración de los usuarios y la valoración rápida.

En la Figura 9 se estudia la relación entre la valoración de los usuarios y la valoración rápida, observándose que tienen una correlación positiva muy débil, ya que prácticamente no existe relación entre las variables.

Una vez descritos los datos de la investigación, se ha diseñado un catálogo de aplicaciones ${ }^{3}$ como recurso para los docentes y las familias, en el cual aparecen las aplicaciones ordenadas según su calificación, además de un enlace con el instrumento de evaluación utilizado. Se considera importante esta tarea, puesto que puede ayudar a muchas familias y docentes a conocer diversas aplicaciones que facilitan el desarrollo de la comunicación de las personas con TEA, así como conocer la calidad de estas. 


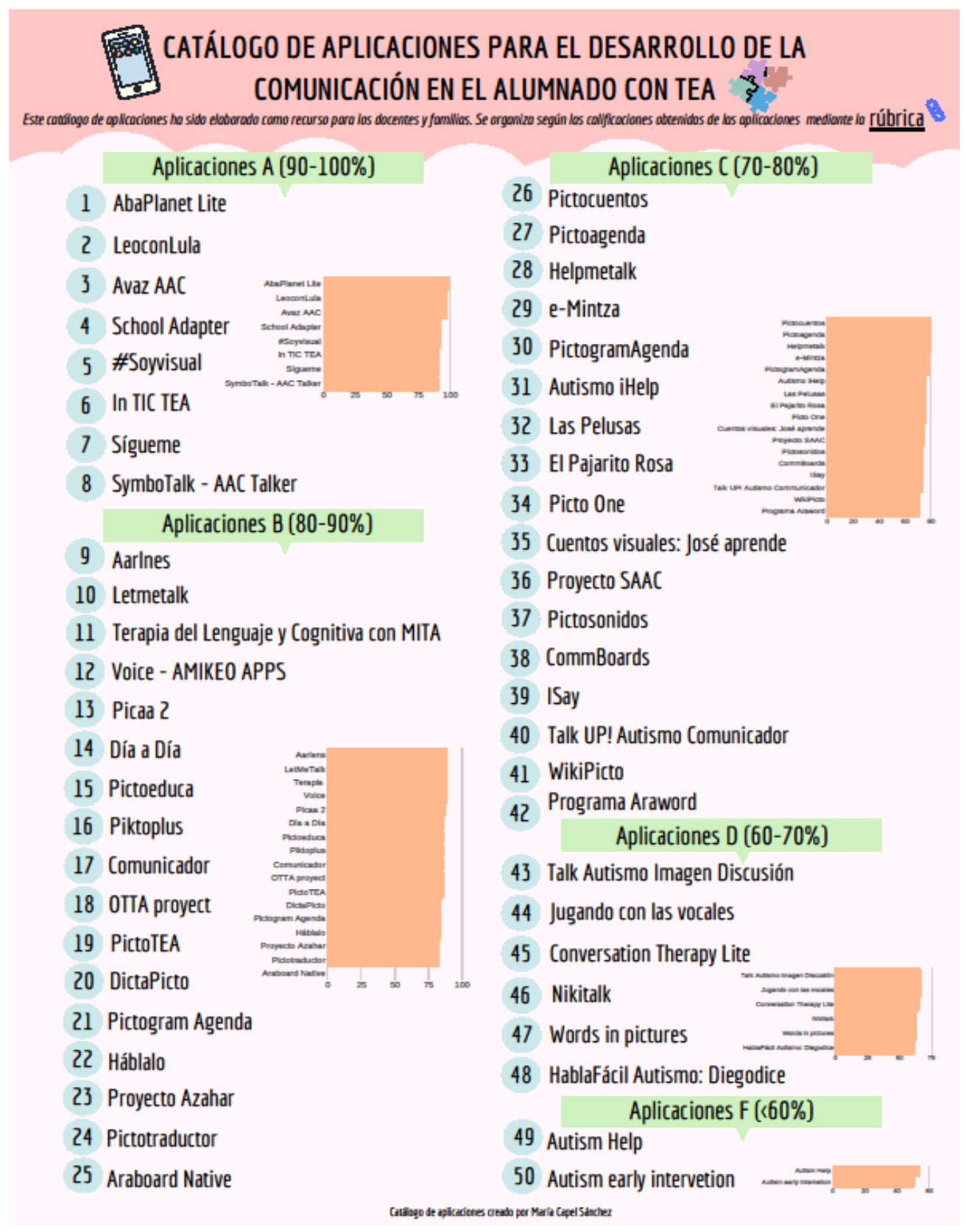

Figura 10. Catálogo de aplicaciones para el desarrollo de la comunicación en el alumnado con TEA 


\section{DISCUSIÓN Y CONCLUSIONES}

Los resultados generales de la investigación se pueden interpretar de la siguiente manera:

Con respecto a los soportes de las aplicaciones, se observa claramente un número muy reducido de aplicaciones de escritorio, en concreto 5 de 50. La explicación de este hecho se da por el gran auge que existe actualmente de los dispositivos móviles y por el interés por desarrollar todo tipo de apps digitales para las personas con TEA (Sanromà et al., 2017). Además, no solo se debe a este aumento, sino también a las características de estos dispositivos móviles. Como bien destacan los autores Mehdipour y Zerehkafi (2013), con estos dispositivos el alumnado puede aprender en cualquier lugar y momento, puesto que el aprendizaje a través del móvil se caracteriza por ser personalizado, individual, en movilidad, ubicuo y portátil. En cambio, el ordenador de mesa puede ser individual y personalizado, pero carece de las demás características.

Para la selección de la muestra se eliminaron las aplicaciones que no fuesen gratuitas, siendo excluidas 44 aplicaciones de 148, por lo que esto indica que hay bastantes aplicaciones que no son de pago. La investigación desarrollada por Larco et al. (2018) destacaba que realizar un estudio con aplicaciones de pago es inviable, añadiendo que muchas familias que tienen hijos que presentan diversidad funcional viven en condiciones de pobreza. Por este motivo, puede ser que los desarrolladores proporcionen más cantidad de aplicaciones gratuitas o en versión Lite que de pago.

De manera general, haciéndose referencia a los resultados obtenidos en la evaluación de las características y diseño técnico de las aplicaciones, se pueden inferir las siguientes explicaciones:

La categoría de Errores y retroalimentación destaca por su valor negativo, por lo que en la gran mayoría de las aplicaciones no se les notifica a los estudiantes de si sus respuestas son correctas e incorrectas y no se les da la respuesta correcta cuando fallan. Que no presenten esta característica es un problema a destacar, debido a que estos automatizarán sus errores. Además, que reciban una retroalimentación positiva, conllevará a un aumento de motivación y éxito en la tarea (Ok et al., 2015).

Otros autores añaden que las aplicaciones destinadas al alumnado con autismo deben incluir refuerzos positivos cuando se acierte o cuando se falle una actividad, es decir, que exista un feedback positivo (Hardy et al., 2002). Por tanto, no se debe obviar esta característica tan relevante en las aplicaciones diseñadas, percibiéndose su inexistencia en casi todas ellas.

Pero no solo se aprecian resultados desfavorables en la categoría anterior, también cabe subrayar las categorías Análisis de errores y Control del progreso con porcentajes muy altos en la calificación más baja. Esto se traduce en que las aplicaciones no contemplan un registro de los tipos de errores que comete el alumno y que no informan de su progreso mediante un sistema de seguimiento. Los estudiantes con TEA necesitan estas características en los 
programas desarrollados para ellos, pues de lo contrario, su aprendizaje será ineficaz y poco significativo.

Según Ok et al. (2015), en ocasiones, los estudiantes cometen errores de forma inconsciente y al azar y, otras veces, los cometen de forma estratégica. Por ello, es importante que la herramienta analice estos datos, determinando si los errores son aleatorios y usándolos para guiar el aprendizaje de los estudiantes. Además, destacan la categoría Control del progreso como la más efectiva en las aplicaciones educativas, debido a que avisa al maestro del rendimiento del alumno y si este ha cumplido o no su objetivo de aprendizaje. Sin esta, el docente debería estar ayudando y supervisando en todo momento al alumno cuando realiza sus actividades en el aula, provocando con esta situación que se sienta excluido.

En diversas investigaciones se menciona que la tecnología de ayuda potencia y aumenta la motivación en los estudiantes. Sanromà et al. (2017) destacan que las TIC presentan una capacidad de motivación, potenciando la atención y disminuyendo los errores en el alumnado. De la misma forma, Cepeda et al. (2017), expone que el uso de estas herramientas facilita al estudiante a tener un aprendizaje más vivencial, conduciendo a una mayor motivación para realizar las tareas.

Según el resultado general de las aplicaciones, la categoría Motivación presenta un $70 \%$ en la calificación más alta, por lo que son aplicaciones que mantienen constantemente la participación de los estudiantes, pero hay un $10 \%$ que expresa que las aplicaciones motivan poco o nada a los estudiantes y un $20 \%$ en las cuales pierden la atención muy rápido. Por lo que, a raíz de esto, se expresa cierto desacuerdo con lo que mencionan los autores, ya que no por utilizar las TIC va a acarrear una gran motivación del alumnado, puesto que esto también dependerá de cómo se adapte la tecnología al alumnado. Es muy importante que la aplicación mantenga el compromiso del estudiante, porque si no, este tendrá muy pocas razones para seguir su aprendizaje.

Por otro lado, se observan resultados muy positivos con respecto a la categoría Estímulos visuales y auditivos. La gran mayoría de estudiantes presentan hiper o hiporreactividad a los estímulos sensoriales o inusual interés en aspectos sensoriales del entorno (APA, 2014). Por ello, es de gran importancia que los desarrolladores hayan tenido en cuenta esta característica, ya que, si el diseño de la aplicación distrae al estudiante, puede conllevar a que no logre su aprendizaje.

En cuanto a las configuraciones personalizadas de las aplicaciones, se aprecia que sus porcentajes están más equilibrados, interpretándose que estas características en el diseño de una aplicación son poco importantes o no tan relevantes. De hecho, en la investigación desarrollada por Larco et al. (2018) concluyeron que la personalización es una de las características que menos se muestra en las aplicaciones evaluadas para las personas con diversidad funcional. Situación que supone una barrera, ya que son aspectos relevantes que pueden ayudar a mejorar la participación de los estudiantes en su proceso de aprendizaje. 
Aunque, por otro lado, sí se puede observar que, en las subcategorías de Vocabulario y gramática y Fotografías, su porcentaje con la máxima puntuación sobresale con respecto a las demás puntuaciones. En las aplicaciones destinadas para el alumnado con TEA, estos aspectos son esenciales, ya que aparte de que cada uno presenta unas características diferentes y deben poder configurarse para cada uno de ellos, el canal visual, es el medio por donde mejor procesan la información. Es por ello que, se han observado un gran número de aplicaciones que permiten usar las imágenes que se deseen o modificar las que la aplicación proporciona. Además, muchas de ellas se basan en sistemas de comunicación visual, donde se relacionan los gráficos y su significado.

El estudio correlacional llevado a cabo en esta investigación conduce a conclusiones similares de otras experiencias de la misma línea de estudio, sin embargo, surgen nuevas explicaciones que no se encuentran en ninguna investigación analizada.

En primer lugar, se estudió la relación de mi valoración rápida como experta tras el análisis previo realizado y la calificación final de las aplicaciones, obteniendo como resultado, una correlación positiva considerable. La explicación sobre la relación de una u otra se debe a que mi valoración como experta puede estar sesgada y condicionada por diversos factores antecedentes a esta. Asimismo, se ha podido ver afectada por las experiencias y estudios consultados sobre evaluación de aplicaciones, además, se han analizado ocho rúbricas y se ha realizado una rúbrica a partir de tres de ellas, por lo que, al echar un vistazo rápido a la aplicación, lo hacía teniendo en cuenta las categorías seleccionadas. Por esto, todo ello ha podido condicionar mi valoración, ya que no es lo mismo valorar de manera rápida la aplicación como una persona que no ha realizado ninguna revisión de bibliografía especializada sobre este tema que como un usuario que no lo ha hecho.

En cambio, los resultados de las variables de la valoración media de los usuarios y la calificación final muestran que no existe ninguna correlación alguna entre ellas. Con estos datos se afirman y verifican las investigaciones donde los autores exponen que la categorización de Apple Store o Google Play de una aplicación como adecuada para el alumnado con diversidad funcional es deficiente, recalcando que deben ser evaluadas de una manera más detallada utilizando una herramienta para precisar su capacidad para apoyar a los estudiantes con NEE. Es por ello, que no debemos seleccionar las aplicaciones al azar sin analizar su calidad educativa o por sus estrellas o comentarios (Ok et al., 2015; Weng y Taber-Doughty, 2015; Bouck et al., 2016; Cepeda et al., 2017; Larco et al., 2018).

Al igual sucede con la relación de la valoración media de los usuarios con mi valoración rápida, aunque existiendo una relación muy débil. Quizás esta pequeña relación se debe a que existe cierta subjetividad en mi valoración rápida, pero sigue estado sesgada por los motivos mencionados anteriormente, por lo que mi valoración no es del todo subjetiva como la de los demás usuarios.

Como bien demuestran los resultados obtenidos, se ha conseguido verificar que, existen aplicaciones digitales educativas de calidad para desarrollar la comunicación en el alumnado con TEA. Como hitos más importantes en relación a los objetivos de este trabajo, se destacan: 
- Se ha conseguido conocer la situación actual del papel que tienen las tecnologías educativas digitales en la intervención educativa en el alumnado con TEA a través de una revisión de bibliografía especializada, observando la falta de investigaciones sobre este tema en el contexto nacional.

- Se ha realizado una rúbrica que evalúa la calidad de las aplicaciones para niños con NEE a partir de tres rúbricas validadas de otros autores. Las rúbricas seleccionadas son de otros países, por lo que esto supone un obstáculo a aquellos docentes que necesitan evaluar con calidad un software y no disponen de documentos de validez científica o rúbricas en su idioma. Por lo que su traducción al castellano ha sido muy útil para aquellos familiares y docentes que no lo dominen. Además, se destaca que hay muy pocos modelos e instrumentos para evaluar la calidad de aplicaciones en nuestro marco contextual, por lo que servirá como un recurso eficaz y funcional.

- Se han seleccionado las aplicaciones que más se adaptan a la investigación, identificando su calidad mediante una rúbrica, obteniendo así, pocas aplicaciones con la máxima calificación. Se considera que el tamaño de la muestra es significante a pesar de excluir 98 aplicaciones en su selección.

- Se ha diseñado y creado un catálogo de aplicaciones que desarrollan la comunicación en el alumnado con TEA con sus calificaciones finales, siendo un gran recurso de ayuda para familias y docentes que se hallan perdidos en este mundo tecnología.

Cabe destacar la gran utilidad de esta investigación por su actualidad en relación a la bibliografía y aplicaciones exploradas y por los recursos que se ofrecen. Debido al gran auge de la tecnología móvil, como profesionales de la educación, debemos estar continuamente actualizados y renovados, contando con un aprendizaje de mejora continua.

\section{REFERENCIAS}

APA (2014). Guía de consulta de los criterios diagnósticos del DSM-5. American Psychiatric Pub.

Bouck, E. C., Satsangi, R. \& Flanagan, S. (2016). Focus on Inclusive Education: Evaluating Apps for Students With Disabilities: Supporting Academic Access and Success. Childhood Education, 92(4), 324-328. https://doi.org/10.1080/00094056.2016.1208014

Boyd, T. K., Hart Barnett, J. E. \& More, C. M. (2015). Evaluating iPad Technology for Enhancing Communication Skills of Children With Autism Spectrum Disorders. Intervention in School and Clinic, 51(1), 19-27. https://doi.org/10.1177/1053451215577476

Cepeda, O., Gallardo, I.M. y Rodríguez, J. (2017). La evaluación de los materiales didácticos digitales. Revista Latinoamericana de Tecnología Educativa (RELATEC), 16(2), 80-95. https://doi.org/10.17398/1695-288X.16.2.79

García, S., Garrote, D. y Jiménez, S. (2016). Uso de las TIC en el Trastorno de Espectro Autista: aplicaciones. Edmetic, 5(2), 134-157. http://hdl.handle.net/10396/14211 
Hardy, C., Ogden, J., Newman, J. \& Cooper, S. (2002). Autism and ICT: A guide for teachers and parents. David Fulton.

Hernández-Sampieri, R., Fernández, C. y Baptista, L. (2014). Metodología de la investigación (6a ed.). McGRAW-HILL.

Higgins, K., Boone, R. \& Williams, D. L. (2000). Evaluating Educational Software for Special Education. Intervention in School and Clinic, 36(2), 109 115. https://doi.org/10.1177/105345120003600207

Jiménez, M.D., Serrano, J.L. y Prendes, M.P. (2017). Estudio de caso de la influencia del aprendizaje electrónico móvil en el desarrollo de la comunicación y el lenguaje con un niño con TEA. Educar, 53(2), 419-443. https://doi.org/10.5565/rev/educar.782

Larco, A., Montenegro, C., Diaz, E. \& Lujan-Mora, S. (2018). Underlying Quality Factors in Spanish Language Apps for People with Disabilities. 2018 International Conference on eDemocracy \& eGovernment (ICEDEG). https://doi.org/10.1109/ICEDEG.2018.8372347

Larco, A., Yanez, C., Almendáriz, V. \& Lujan-Mora, S. (2018). Thinking about inclusion: Assessment of multiplatform apps for people with disability. 2018 IEEE Global Engineering Education

Conference (EDUCON). https://doi.org/10.1109/educon.2018.8363250

Marquès, P. (1999). Criterios para la clasificación y evaluación de espacios web de interés educativo. Educar (25), 95-111. https://www.raco.cat/index.php/Educar/article/view/20717

McNaughton, D. \& Light, J. (2013). The iPad and Mobile Technology Revolution: Benefits and Challenges for Individuals who require Augmentative and Alternative Communication. Augmentative and Alternative Communication, 29(2), 107-116. https://doi.org/10.3109/07434618.2013.784930

Mehdipour, Y. \& Zerehkafi, H. (2013). Mobile Learning for Education: Benefits and Challenges. International Journal of Computational Engineering Research, 3(6), 93-101. http://www.ijceronline.com/papers/Vol3_issue6/part\%203/P03630930100.pdf

Montero, G. (2019). Guía para el profesorado. Uso responsable y seguro de las TIC en el alumnado con trastorno del espectro del autismo. Confederación Autismo España.

More, C. M., \& Travers, J. C. (2013). What's app with that? Selecting educational apps for young children with disabilities. Young Exceptional Children, 16(2), 15-32. https://doi.org/10.1177/1096250612464763

Ok, M. W., Kim, M. K., Kang, E. Y. \& Bryant, B. R. (2015). How to Find Good Apps: An Evaluation Rubric for Instructional Apps for Teaching Students With Learning Disabilities. 


Intervention in School and Clinic, 51(4), 244-
252. https://doi.org/10.1177/1053451215589179

Pastor, C. A., Sánchez, J. M. y Zubillaga, A. (2014). Diseño Universal para el aprendizaje (DUA). https://www.educadua.es/doc/dua/dua_pautas_intro_cv.pdf

Salinas, P. (2012). Metodología de la investigación científica. Universidad de Los Andes.

Sanromà, M., Lázaro, J.L. y Gisbert-Cervera, M. (2017). La tecnología móvil. Una herramienta para la mejora de la inclusión digital de las personas con TEA. Psicología, Conocimiento y Sociedad, 7(2), 227-251. http://www.scielo.edu.uy/pdf/pcs/v7n2/1688-7026-pcs-702-00173.pdf

Terrazas, M., Sánchez, S. y Becerra, M.T. (2016). Las TIC como herramienta de apoyo para personas con Trastorno del Espectro Autista (TEA). Revista nacional e internacional de educación inclusiva, 9(2), 102-136. https://dialnet.unirioja.es/servlet/articulo?codigo=5600282

Walker, H. (2010). Evaluation rubric for iPod apps. https://learninginhand.com/blog/evaluation-rubric-for-educational-apps.html

Weng, P.L. \& Taber-Doughty, T. (2015). Developing an App Evaluation Rubric for Practitioners in Special Education. Journal of Special Education Technology, 30(1), 4358. https://doi.org/10.1177/016264341503000104

Wing, L. (1998): El autismo en niños y adultos. Una guía para la familia. Paidós.

\section{Para citar este artículo:}

Capel Sánchez, M. (2021). Análisis y evaluación de aplicaciones para desarrollar la comunicación en el alumnado con trastorno del espectro autista. Edutec. Revista Electrónica De Tecnología Educativa, (75), 168-187. https://doi.org/10.21556/edutec.2021.75.1681 